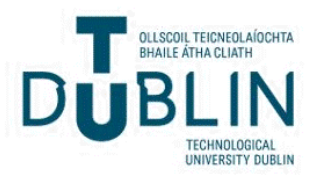

Technological University Dublin ARROW@TU Dublin

\section{Reconfigurable Axial Ratio in Compact GNSS Antennas}

\author{
Adam Narbudowicz \\ Technological University Dublin, adam.narbudowicz@mydit.ie
}

Follow this and additional works at: https://arrow.tudublin.ie/ahfrcart

Part of the Engineering Commons

\section{Recommended Citation}

Narbudowicz, A., Ammann, M. \& Heberling, D. (2016). Reconfigurable Axial-Ratio in Compact GNSS

Antennas. IEEE Xplore Digital Library2016. doi:10.1109/TAP.2016.2589975.

This Article is brought to you for free and open access by the Antenna \& High Frequency Research Centre at ARROW@TU Dublin. It has been accepted for inclusion in Articles by an authorized administrator of ARROW@TU Dublin. For more information, please contact arrow.admin@tudublin.ie, aisling.coyne@tudublin.ie, gerard.connolly@tudublin.ie.

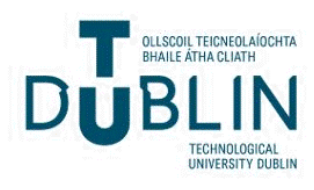




\section{Reconfigurable Axial-Ratio in Compact GNSS Antennas}

\author{
A. Narbudowicz, M. J. Ammann and D. Heberling
}

\begin{abstract}
A new adaptive technique for steering the direction of minimum axial-ratio $(<1 \mathrm{~dB})$ towards the reflected or jamming signal is proposed for GNSS antennas. It relies on simple, low-cost commercially-available circularly-polarized antenna elements without influencing their gain. Its performance is demonstrated using two different antenna geometries, with five different configurations. The measurements agree well with simulation and theoretical calculations. The technique allows a combined $1 \mathrm{~dB}$ axial-ratio beamwidth of up to $83^{\circ}$.
\end{abstract}

Index Terms-GPS, GNSS, Circular polarization, Axial ratio, Reconfigurable antenna, Adaptive antenna.

\section{INTRODUCTION}

CIRCULARLY-polarized signals have been exploited for many applications due to their capability to suppress reflected signals. This property is most useful in the Global Navigation Satellite Systems (GNSS), where cross-polarized signals reflected by high-rise buildings (e.g. 'urban canyons') degrade positioning accuracy [1]. Good suppression of undesired reflected signals demands high circular-polarization (CP) purity, indicated by a low axial-ratio (AR). Obtaining an $\mathrm{AR} \leq$ $1 \mathrm{~dB}$ over wide beamwidth for planar antennas is still a challenging task, however achievable by $3 \mathrm{D}$ antenna structures (e.g. [2, 3]). Some techniques for planar patches allow $\sim 0 \mathrm{~dB}$ $\mathrm{AR}$ at boresight, however do not discuss beamwidth performance [4]. In this communication, a novel technique for AR reconfiguration, is proposed, which, rather than using expensive and complex broadbeam AR antennas, relies on basic antenna shapes to reconfigure AR without impacting the dominant polarization gain or radiation pattern.

Simple CP antennas - e.g. microstrip patches - exhibit very good AR at boresight, which gradually degrades towards the horizon. Many antennas were designed to allow broadbeam AR [2, 3, 5 - 11]. Most notable is the quadrifilar-helix $(\mathrm{QH})$ antenna, which uses four conducting helices, wrapped around a common axis and fed with sequential $90^{\circ}$ phase difference. A design proposed in [4] offers an $\mathrm{AR}<3 \mathrm{~dB}$ over the beamwidth of approximately $160^{\circ}$. QH-inspired antennas [5, 6] offer $130^{\circ}$

This paragraph of the first footnote will contain the date on which you submitted your paper for review. This work was supported by Irish Research Council under "ELEVATE: Irish Research Council International Career Development Fellowship - co-funded by Marie Cure Actions", grant no. ELEVATEPD/2014/79.

A. Narbudowicz is jointly with Antenna and High Frequency Research Centre, Dublin Institute of Technology, Dublin, Ireland and Institute of High Frequency Technology, RWTH Aachen University, Germany.

M. J. Ammann is with Antenna and High Frequency Research Centre, Dublin Institute of Technology, Dublin, Ireland.

D. Heberling is with Institute of High Frequency Technology, RWTH Aachen University, Germany. and $80^{\circ}$ respectively. Antennas proposed for RFID readers in $[7,8]$ have respective AR beamwidths of $163^{\circ}$ [7] and $60^{\circ}$ [8]. A stacked patch antenna in [9] is reported to provide up to $180^{\circ}$ of $3 \mathrm{~dB}$ AR beamwidth. A cavity-backed spiral-slot [10] allows $215^{\circ}$. In [11] a technique was proposed for a planar antenna with broad beam AR of up to $126^{\circ}$. This is achieved by appropriately combining four dipole antennas, i.e. by adjusting their orientation, distance and phase shift. Despite the broad beam $\mathrm{AR}$ and low profile of $0.8 \mathrm{~mm}$, the antenna has a bidirectional radiation pattern, producing LHCP to the front and RHCP to the rear. However for GNSS applications, this pattern would receive ground-reflected LHCP signals, thus decreasing positioning accuracy.

Despite the performance presented [2-11], most commercial GNSS devices use simple antennas, e.g. CP patches [12 - 14], due to their low cost and miniaturization capabilities (some commercial GPS patch antennas are as small as $12 \times 12 \mathrm{~mm}$ [14]). However, their AR degrades away from boresight.

This communication proposes a novel and simple methodology to adjust the direction of the optimum axial-ratio $(<1 \mathrm{~dB})$, without significantly changing the power pattern of the dominant circular polarization. The technique is intended for any state-of-the-art dual-fed CP antennas or dual linearlypolarized antennas, most notably for basic antennas such as microstrip patches. It is a common belief, that $\mathrm{CP}$ antennas offer optimum performance when their two orthogonal components are fed with $90^{\circ}$ phase shift. This is however true only for boresight radiation and was not deeply studied in the literature. The only exception is [15] where some early theoretical studies (without experimental validation) are presented for two idealized dipoles. By using the proposed method, one can synthesize an adaptive AR pattern, which can provide an $\mathrm{AR} \leq$ $1 \mathrm{~dB}$ within a $83^{\circ}$ beamwidth. The system is intended to improve GNSS performance in rich multipath environments, where undesired cross-polarized signals arrive from various directions. However other applications are also suitable, such as direct modulation systems [16] or rotation sensors [17].

\section{PRINCIPLE OF OPERATION}

Let us assume there are two linear radiating sources, represented as vectors $v_{l}$ and $v_{2}$, which intersect at an angle $\alpha \epsilon$ $\left(0^{\circ} ; 180^{\circ}\right)$. The vectors are located in the $x y$-plane, in the coordinate system depicted in Fig. 1.

For the this case, the far field can be approximated as a sphere

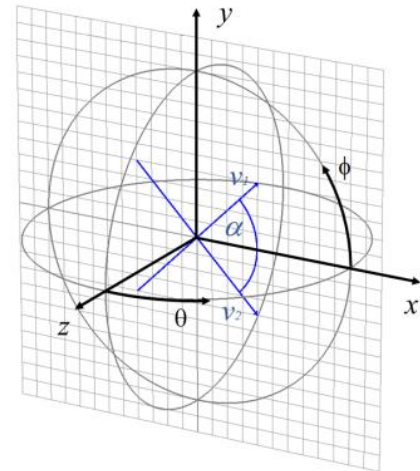

Fig. 1. Visualization of vectors $v_{l}, v_{2}$ and angle $\alpha$ between them in the spherical coordinate system. 
$\mathrm{S}_{\mathrm{ff}}$ of a radius much greater than the length of either $v_{1}$ or $v_{2}$. The electric (and magnetic) fields generated by $v_{1}$ and $v_{2}$ are tangential to $S_{\mathrm{ff}}$. Therefore the angle $\alpha$ formed by the vectors $v_{I}$ and $v_{2}$ will be seen in the farfield as a projection $\alpha^{\prime}(\phi, \theta)$, i.e. a projection of $\alpha$ onto a plane tangential to the sphere $S_{\mathrm{ff}}$ at a given $\theta$ and $\phi$. In general, this angle $\alpha^{\prime}$ has a different value to $\alpha$. Only the projection at directions along the $z$-axis will produce $\alpha^{\prime}\left(\theta=0^{\circ}\right)=\alpha^{\prime}\left(\theta=180^{\circ}\right)=\alpha$, therefore most $\mathrm{CP}$ antennas would use $\alpha=90^{\circ}$ to achieve good performance along the $z$-axis. In general however, the angle $\alpha^{\prime}$ is dependent on $\theta$ and $\phi$. For the two principal cuts - $x z$-plane $\left(\phi=0^{\circ}\right.$,) and $y z$ plane $\left(\phi=90^{\circ}\right)$, the angle $\alpha^{\prime}$ can be calculated using equations:

$$
\begin{aligned}
& \alpha_{\phi=0^{\circ}}=2 \operatorname{artn}\left(\frac{\operatorname{tn}\left(\frac{\alpha}{2}\right)}{\cos (\theta)}\right) \\
& \alpha_{\phi=90^{\circ}}^{\prime}=2 \operatorname{artn}\left(\frac{\operatorname{ctn}\left(\frac{\alpha}{2}\right)}{\cos (\theta)}\right)
\end{aligned}
$$

Eq. (1a) indicates, that for $\alpha>90^{\circ}$ there is a point in the horizontal plane, for which $\alpha^{\prime}=90^{\circ}$. On the contrary, according to (1b) if the angle $\alpha<90^{\circ}$ there is a point in the elevation plane, where $\alpha^{\prime}=90^{\circ}$. The necessary (but not sufficient) condition to achieve $\mathrm{CP}$ at a given $\theta$ and $\phi$ is $\alpha^{\prime}=90^{\circ}$, i.e. the angle as seen in the far field - not $\alpha$ itself. This implies that one could try to control and direct the optimum AR for a desired $\theta$ and $\phi$ by changing the angle $\alpha$. This solution is obviously highly

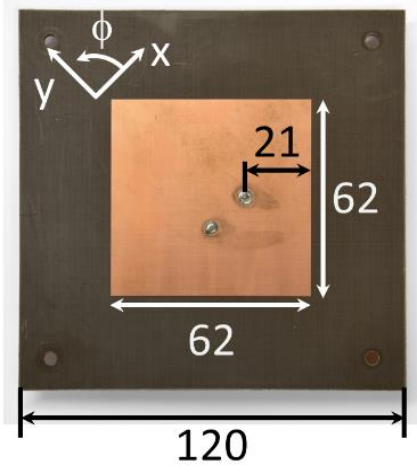

a)

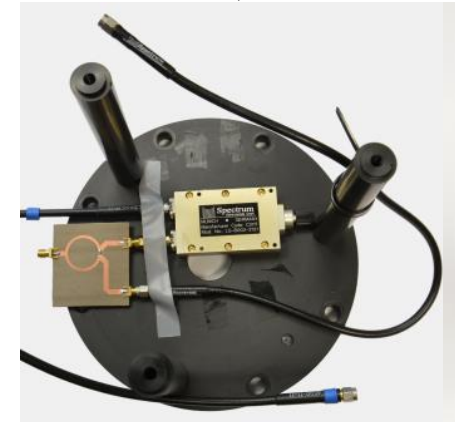

c)

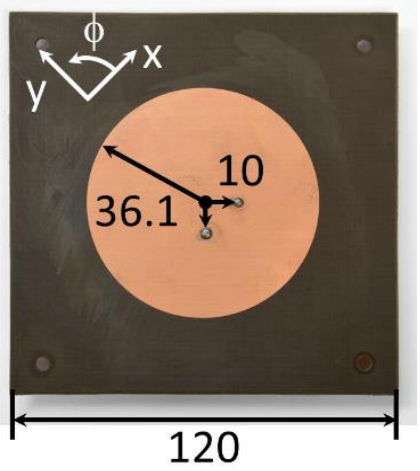

b)

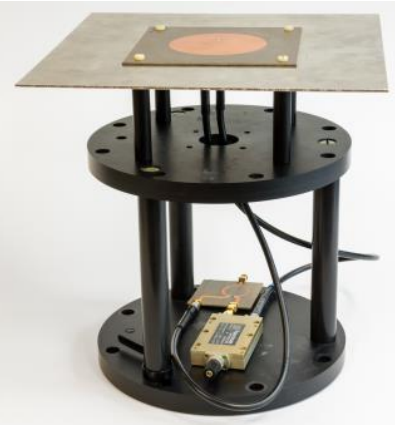

d)
Fig. 2. Experimental setting used as a proof of concept (all dimensions in $\mathrm{mm}$ ): a) rectangular patch antenna; b) circular patch antenna; c) phase shifting circuitry with antenna holder; d) full setup with aluminium sheet.

impractical, as physically changing $\alpha$ would require mechanical actuators. Instead, the proposed solution keeps a constant $\alpha=90^{\circ}$ and adjusts the phase shift $\Delta_{p h}$ between the two orthogonal feeds. This is demonstrated in the following section to produce an effect equivalent to changing $\alpha$, i.e. allows adjustment of the direction of minimum AR.

Practical antennas are not a point source, but rather integrate the contribution of the electric fields from the full aperture, thus making the exact analysis using the above method more challenging. Nevertheless, it will be demonstrated both numerically and experimentally that by only adjusting the phase shift $\Delta_{\mathrm{ph}}$ between two orthogonal components, a good AR $<1$ $\mathrm{dB}$ can be produced for various angles away from boresight.

\section{PROOF OF CONCEPT}

The concept was tested using two simple CP patch antennas shown in Fig. 2, one rectangular and one circular, with the optimum $S_{11}$ at 1.575 and $1.590 \mathrm{GHz}$ respectively. The antennas were prototyped on Taconic ${ }^{\mathrm{TM}}$ TLY -5 substrate of $1.5 \mathrm{~mm}$ thickness and $\varepsilon_{r}=2.2$. To vary the phase, a configuration using a Wilkinson power divider and an adjustable phase shifter [18] as seen in Fig. 2c was used. The pattern measurements were made using a spherical near-field method in the anechoic chamber of RWTH Aachen University. Before each measurement the phase at each cable feed was measured using vector network analyzer, demonstrating a phase error (at cable terminals) less than $0.1^{\circ}$. The patch was backed by a $240 \times 240$ $\mathrm{mm}$ flat aluminium sheet. This reduced the angular range over which AR can be controlled, but was necessary to mitigate the influence of anechoic chamber's positioner. This is solely to allow accurate measurement and the core principle can be applied to patches with smaller groundplane size.

The S-parameters are shown in Fig. 3. It can be seen, that

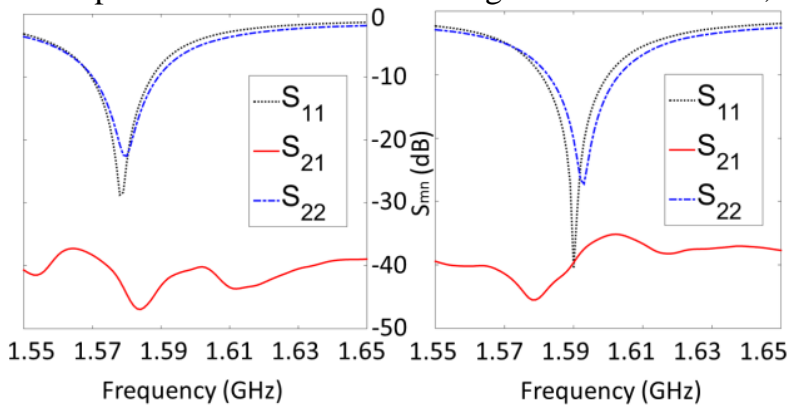

a)

b)

Fig. 3. Measured S-parameters of the two antennas: a) rectangular; b) circular. both antennas exhibit $\sim 40 \mathrm{~dB}$ isolation between ports, ensuring the excitation phase at one port does not influence the other. The centre frequencies are $1.575 \mathrm{GHz}$ for the rectangular patch and $1.59 \mathrm{GHz}$ for the circular. All subsequent results are be shown for these frequencies.

The AR was measured with five different phase shifts $\Delta_{p h}$ : $78^{\circ}, 84^{\circ}, 90^{\circ}, 96^{\circ}$ and $102^{\circ}$. This corresponds to $\pm 6^{\circ}$ steps around the traditionally used $90^{\circ}$. The values are chosen, as $6^{\circ}$ phase inaccuracy is expected to degrade the AR at boresight $\left(\theta=0^{\circ}\right)$ by $\sim 1 \mathrm{~dB}$.

Table I compares the simulated and measured values of directions of minimum AR for both antennas. It can be seen, that the maximum error between simulated and measured values is $13^{\circ}$ (for rectangular patch at $\Delta_{p h}=102^{\circ}$ ) with a mean 
TABLE I

DIRECTION OF AR MINIMA

\begin{tabular}{ccccc}
\hline \hline & \multicolumn{4}{c}{$\Delta_{\mathrm{ph}}$} \\
\cline { 2 - 5 } & \multicolumn{2}{c}{$78^{\circ}$} & $84^{\circ}$ & \multicolumn{2}{c}{$96^{\circ}$} & $102^{\circ}$ \\
& \multicolumn{2}{c}{ In $x z$-plane } & \multicolumn{2}{c}{ In $y z$-plane } \\
\hline Rectangular: & & & & \\
simulated & $-32^{\circ}$ & $-20^{\circ}$ & $-27^{\circ}$ & $-43^{\circ}$ \\
& $+36^{\circ}$ & $+23^{\circ}$ & $+22^{\circ}$ & $+34^{\circ}$ \\
measured & $-30^{\circ}$ & $-20^{\circ}$ & $-17^{\circ}$ & $-30^{\circ}$ \\
Circular: & $+33^{\circ}$ & $+15^{\circ}$ & $+21^{\circ}$ & $+30^{\circ}$ \\
simulated & $-31^{\circ}$ & -20 & $-26^{\circ}$ & $-39^{\circ}$ \\
& $+31^{\circ}$ & $+20^{\circ}$ & $+20^{\circ}$ & $+31^{\circ}$ \\
measured & $-29^{\circ}$ & -20 & $-22^{\circ}$ & $-28^{\circ}$ \\
& $+25^{\circ}$ & $+14^{\circ}$ & $+19^{\circ}$ & $+26^{\circ}$ \\
\hline \hline
\end{tabular}

absolute error of only $4.7^{\circ}$.

Figs. 4 and 5 compare the simulated and measured AR performance of the rectangular and circular antennas respectively. The results are shown for antenna center frequencies, i.e. $1.575 \mathrm{GHz}$ for rectangular and $1.59 \mathrm{GHz}$ for the circular patch. For both designs a clear trend can be seen: small variations around the phase shift $\Delta_{p h}=90^{\circ}$ can broaden the AR beamwidth in one plane (and shift the direction of minimum axial ratio) at the cost of narrowing it in the orthogonal plane: for $\Delta_{p h}<90^{\circ}$ the minima move in the $x z$ plane, while for $\Delta_{p h}>90^{\circ}$ the move in the $y z$-plane. This is true for all configurations, where the $y z$-plane passes between the patch feeds (and the center of the patch). Although for fixed $\Delta_{p h}$ the improvement in one plane demands a degradation in the other, this can be overcome by using multiple transceivers each executing different phase shifts, or state-of-the-art digital beamforming techniques. With decreasing cost and size of RF

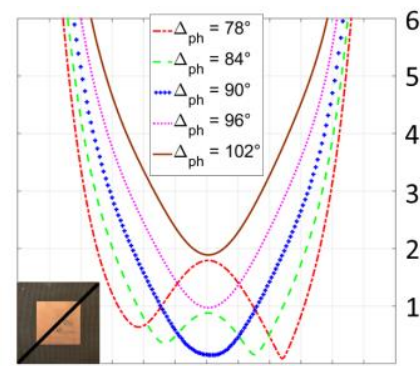

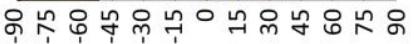
Angle $\theta$ (deg)

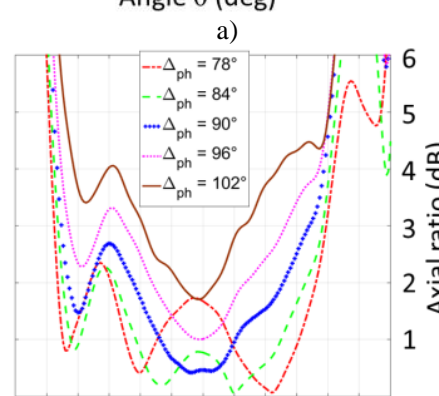

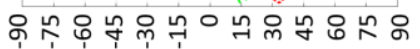
Angle $\theta$ (deg)

c)

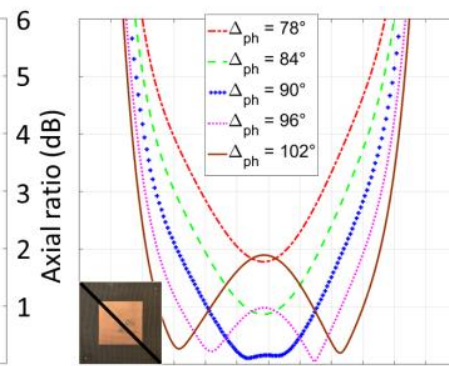

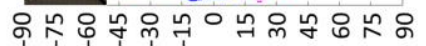
Angle $\theta$ (deg)

b)

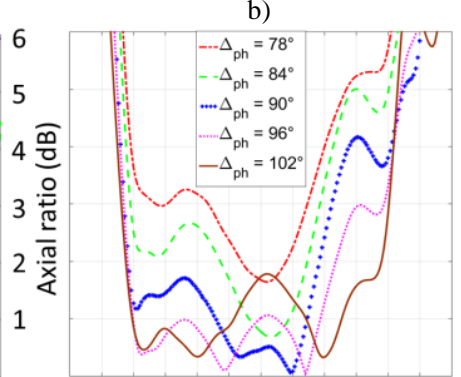

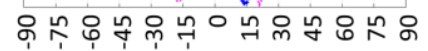
Angle $\theta$ (deg)

d)
Fig. 4. Measured and simulated axial-ratio of the rectangular patch for two principal cuts: a) simulated $x z$-plane; b) simulated $y z$-plane; c) measured $x z$ plane; d) measured $y z$-plane. Insets in the figures demonstrate the plane, for which the plot is shown. frontends, this option will become more attractive over time.

The AR curves are principally symmetric with respect to $\theta=0^{\circ}$, which is a direct consequence of the patch symmetry and is echoed in Eqn (1a) and (1b). Regrettably, the proposed technique does not allow producing a single non-mirrored AR minimum.

In the measured data an additional peak can be seen around $\theta=-45^{\circ}$ for both rectangular and circular patches in Figs 4(c), (d) and 5(c), (d). This is an artificial effect, due to LHCP backlobe reflected from the positioner structure, also housing the phase-shifting circuitry. This effect was mitigated for the main directions by installing a $240 \times 240 \mathrm{~mm}$ aluminum screen, which however does not shield wider angles (i.e. $|\theta|>45^{\circ}$ ).

A slight difference between the phases of the two orthogonal modes in the patch can be seen as a flat area around boresight for $\Delta_{p h}=90^{\circ}$.

For the rectangular patch, an $\mathrm{AR}<1 \mathrm{~dB}$ is achieved in $x z$ plane from $-37^{\circ}$ to $-22^{\circ}$ and $14^{\circ}$ to $46^{\circ}\left(\Delta_{p h}=78^{\circ}\right)$, and $-32^{\circ}$ to $+37^{\circ}\left(\left(\Delta_{p h}=84^{\circ}\right)\right.$ as seen in Fig 4. In the $y z$-plane the peak below $\theta<-45^{\circ}$ can be ignored as a measurement artefact, hence

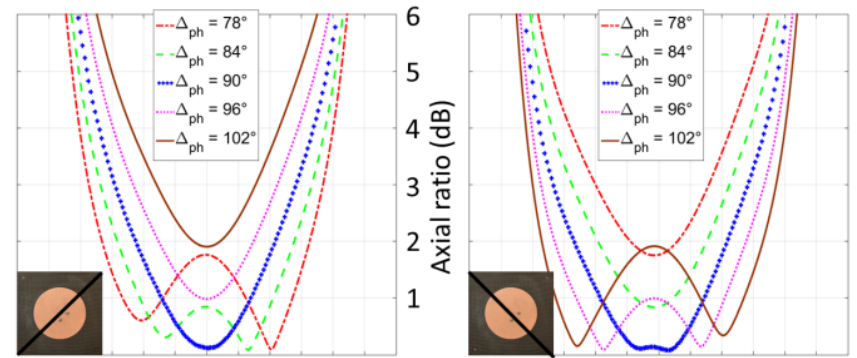

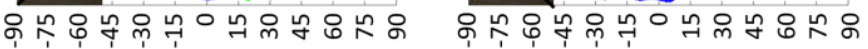
Angle $\theta$ (deg)

a)

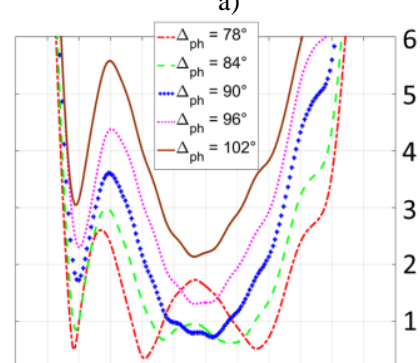

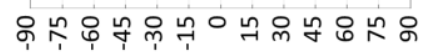
Angle $\theta$ (deg)

c)

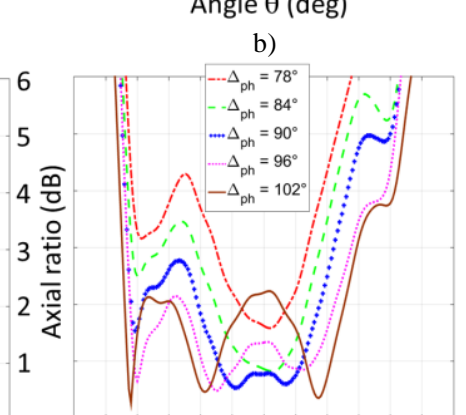

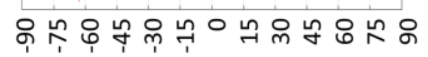
Angle $\theta$ (deg)

d)

Fig. 5. Measured and simulated axial-ratio of the circular patch for two principal cuts: a) simulated $x z$-plane; b) simulated $y z$-plane; c) measured $x z$ plane; d) measured $y z$-plane. Insets in the figures demonstrate the plane, for which the plot is shown.

$\mathrm{AR}<1 \mathrm{~dB}$ is achieved from $-45^{\circ}$ to $-13^{\circ}$ and $+22^{\circ}$ to $+38^{\circ}\left(\Delta_{p h}\right.$ $=102^{\circ}$ ), and from $-45^{\circ}$ to $+26^{\circ}$ with a small peak below $1.1 \mathrm{~dB}$ at $4^{\circ}\left(\Delta_{p h}=96^{\circ}\right)$. For the circular patch (Fig 5) respective values are: in $x z$-plane from $-35^{\circ}$ to $-20^{\circ}$ and $+13^{\circ}$ to $+33^{\circ}\left(\Delta_{p h}=78^{\circ}\right)$ and from $-25^{\circ}$ to $+24^{\circ}\left(\Delta_{p h}=84^{\circ}\right)$; in $y z$-plane from $-34^{\circ}$ to $-22^{\circ}$ and $+20^{\circ}$ to $+32^{\circ}\left(\Delta_{p h}=102^{\circ}\right)$, and from $-29^{\circ}$ to $-12^{\circ}$ and $+9^{\circ}$ to $23^{\circ}\left(\Delta_{p h}=96^{\circ}\right)$.

Fig. 6 shows the simulated $S_{11}$ for various $\Delta_{\mathrm{ph}}$ (with lossless power divider), demonstrating proposed method has negligible impact on $\mathrm{S}_{11}$. 
Fig. 7 shows the measured Right-Hand CP (RHCP) realized gain patterns (including phase shifter losses). It can be seen, that

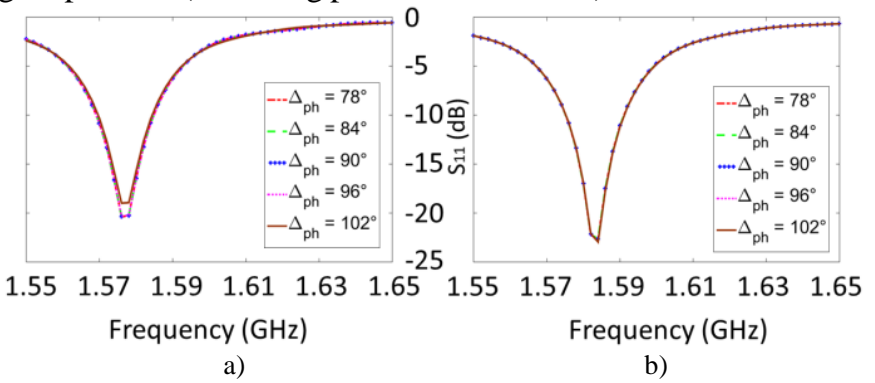

Fig. 6. Simulated $\mathrm{S}_{11}$ for various $\Delta_{p h}$ : a) rectangular patch; b) circular patch. the all curves strictly follow each other, demonstrating that the proposed method has no significant impact on the gain or radiation pattern of the dominant (RHCP) polarization. The maximum gain at boresight is $5.5-5.65 \mathrm{dBic}$. It was demonstrated in Fig. 3 that the isolation between ports is 40 $\mathrm{dB}$. This means that the phase shift at one port will not impact the reflection coefficient at the other port, i.e. that the $\mathrm{S}$ parameters are not dependent on $\Delta_{p h}$.

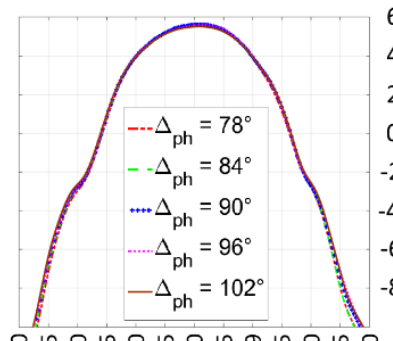

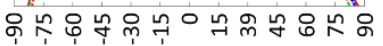
Angle $\theta$ (deg)

a)

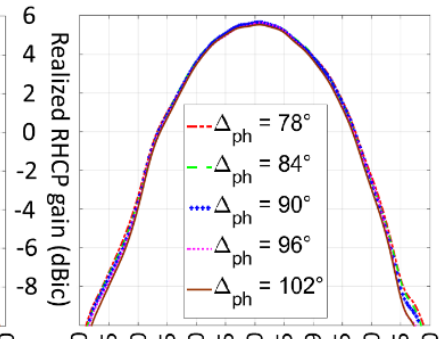

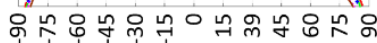
Angle $\theta$ (deg)

b)
Fig. 7. Measured RHCP realized gains of the circular patch: a) $\phi=0^{\circ}$ plane; b) $\phi=0^{\circ}$ plane.

When combining all configurations, the $1 \mathrm{~dB}$ AR beamwidth can be achieved for the rectangular patch from $-37^{\circ}$ to $+46^{\circ}\left(83^{\circ}\right.$ span in $x z$-plane) and from $-45^{\circ}$ to $+38\left(83^{\circ}\right.$ span in yz-plane $)$. Respective values for the circular patch are from $-35^{\circ}$ to $+33^{\circ}$ $\left(68^{\circ}\right.$ span in $x z$-plane $)$ and $-32^{\circ}$ to $+32^{\circ}\left(66^{\circ}\right.$ span in $y z$-plane $)$.

\section{CONCLUSION}

To conclude our findings, it is demonstrated that the best phase shift of $\mathrm{CP}$ antenna is not necessarily $90^{\circ}$, but rather is dependent on the signal's direction of arrival. This can be implemented for a low-cost method to adaptively improve the AR performance, while using basic off-the-shelf GNSS antennas. Based on the values reported in Table I, if the incoming signal arrives from around $\theta=30^{\circ}$ (for investigated antennas), the best cross-polar discrimination is achieved with $\Delta_{p h}=78^{\circ}$ (for $\phi$ values closer to $0^{\circ} / 180^{\circ}$ ) and $\Delta_{p h}=102^{\circ}$ (for $\phi$ values closer to $90^{\circ} / 270^{\circ}$ ). For $\theta=20^{\circ}$ the corresponding values are $\Delta_{p h}=82^{\circ}$ and $\Delta_{p h}=96^{\circ}$. These values however are antenna specific. Most notably it is considered, that an antenna with a smaller ground plane will allow AR steering within an even wider beamwidth (results not shown for brevity).

The method, although being general, is considered to be most useful for global navigation applications, where one has an initial estimation of the signal angle-of-arrival, simplifying the switching algorithm and enabling an optimal AR pattern. With the advancement of digital beamforming and software defined radio, one can generate the necessary phase shifts in the digital domain, opening up a plethora of new applications for the proposed technique (e.g. multiple AR configurations can be generated simultaneously).

\section{REFERENCES}

[1] W. Alshrafi, U. Engel, and T. Bertuch, "Compact controlled reception pattern antenna for interference mitigation tasks of global navigation satellite system receivers", IET Microwaves, Antennas \& Propagation, vol. 9 (6), pp. 593-601, Apr. 2015.

[2] S. Gao, Qi Luo, and Fuguo Zhu, Circularly Polarized Antennas. WileyIEEE Press, 2014

[3] J. Rabemanantsoa, and A. Sharaiha, "Size Reduced Multi-Band Printed Quadrifilar Helical Antenna," IEEE Transactions on Antennas and Propagation, vol. 59 (9), pp. 3138-3143, Sep. 2011.

[4] S. Ogurtsov, and S. Koziel, "Enhancement of circular polarization quality of single-patch two-input microstrip antennas," Journ. of Electromagnetic Waves and Applications, vol. 30 (6), pp. 767-779, Apr. 2016.

[5] Kejia Ding, Yazhou Wang, and Xiaojun Xiong, "A Novel Wide-Beam Circularly Polarized Antenna for SDARS Applications," IEEE Antennas and Wireless Propagation Letters, vol. 11, pp. 811-813, Jul. 2012.

[6] Changjiang Deng, Yue Li, Zhijun Zhang, and Zhenghe Feng, "A Circularly Polarized Pattern Diversity Antenna for Hemispherical Coverage," IEEE Transactions on Antennas and Propagation, vol. 62 (10), pp. 5365-5369, Oct. 2014.

[7] Hae-Won Son, Hyuk Park, Kyong-Hee Lee, Gwang-Ja Jin, and MoonKyun Oh, "UHF RFID Reader Antenna With a Wide Beamwidth and High Return Loss," IEEE Transactions on Antennas and Propagation, vol. 60 (10), pp. 4928-4932, Oct. 2012.

[8] R. Caso, A. Michel, M. Rodriguez-Pino, and P. Nepa, "Dual-Band UHFRFID/WLAN Circularly Polarized Antenna for Portable RFID Readers," IEEE Trans. on Ant. and Prop., vol. 62 (5), pp. 2822-2826, May. 2014.

[9] X. L. Bao, and M. J. Ammann, "Dual-frequency dual circularly-polarised patch antenna with wide beamwidth," Electronics Letters, vol. 44 (21), pp. 1233-1234, Oct. 2008.

[10] X. L. Bao, and M. J. Ammann, "A Cavity-Backed Spiral Slot Antenna with Wide Axial Ratio Beamwidth for GPS System," Microwave and Optical Technology Letters, vol. 56 (5), pp. 1050-1054, May 2014.

[11] Yu Luo, Qing-Xin Chu, and Lei Zhu, “A Low-Profile Wide-Beamwidth Circularly-Polarized Antenna via Two Pairs of Parallel Dipoles in a Square Contour," IEEE Transactions on Antennas and Propagation, vol. 63 (3), pp. 931-936, Mar. 2015.

[12] Nasimuddin, Xianming Qing, and Zhi Ning Chen, "A Compact Circularly Polarized Slotted Patch Antenna for GNSS Applications," IEEE Trans. on Ant. and Prop., vol. 62 (12), pp. 6506-6509, Dec. 2014.

[13] Nasimuddin, Zhi Ning Chen, and Xianming Qing, "Slotted Microstrip Antennas for Circular Polarization with Compact Size," IEEE Antennas and Propagation Magazine, vol. 55 (2), pp. 124-137, Apr. 2013.

[14] Specification: GP.1575.12.4.A.02, Taoglas Ltd., 2015. Available: http://www.taoglas.com/antennas/GPS-GLONASS_Antennas/ Internal_GPS__Passive_Ceramic_Patch_Antennas/

[15] A. Narbudowicz, M. J. Ammann, and J. Przewocki, "Shaping the AxialRatio Footprint of Crossed-Dipole Antennas," in Proc. Loughborough Antennas and Propagation Conference (LAPC), Loughborough, UK, Nov. 2014, pp. 598-601.

[16] M. Amin, S. Ahmed, V. Fusco, H. Cantu and T. Ratnarajah, "The Effect of Spatial Axial Ratio Variation on QPSK Modulation Encoded Using Orthogonal Circularly Polarized Signals," in Proc. European Conference on Wireless Technologies, Munich, Germany, Oct. 2007, pp. 62-65.

[17] V. Sipal, A. Narbudowicz, and M. J. Ammann, "Contactless Measurement of Angular Velocity Using Circularly Polarized Antennas," IEEE Sensors Journal, vol. 15 (6), pp. 3459-3466, June 2015.

[18] Phase Adjusters with Ball Bearing Support, Spectrum Elektrotechnik GmbH, Munich, Germany, 2015. Available: http://www.spectrumet.org/NEW WEB2/images/additional-information/LS-B002-2121.pdf 